\title{
Efficient procedure to remove ECG from sEMG with limited deteriorations: Extraction, quasi-periodic detection and cancellation
}

\author{
François Nougarou ${ }^{\mathrm{a}, *}$, Daniel Massicotte ${ }^{\mathrm{a}}$, Martin Descarreaux ${ }^{\mathrm{b}}$ \\ a Department of Electrical and Computing Engineering, University of Québec at Trois-Rivières, Québec, Canada

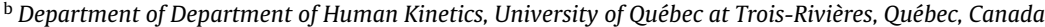

\section{A R T I C L E I N F O}

\section{Article history:}

Received 1 August 2016

Received in revised form 16 June 2017

Accepted 20 July 2017

Available online 4 August 2017

\section{Keywords:}

ECG cancellation

Quasi-periodic signals detection

Discrete wavelet transforms

Independent component analysis

\begin{abstract}
A B S T R A C T
The interpretations of the surface electromyography (sEMG) signals from the trunk region are strongly distorted by the heart activity (ECG), especially in case of low-amplitude EMG responses analyses. Many methods have been investigated to resolve this nontrivial problem, by using advanced data processing on the overall sEMG recorded signal. However, if they reduce ECG artifacts, those cancellation methods also deteriorate noiseless parts of the signal. This work proposes an original ECG cancellation method designed to limit the deterioration of sEMG information. To do that, the proposed techniques does not directly attempt to remove the ECG, but is based on two main steps: the localization of ECG and the cancellation of ECG but only where heart pulses have been detected. The phase of localization efficiently extracts the ECG contribution by combining the discrete wavelet transforms (DWT) and the method of independent component analysis (ICA). And finally, this phase takes advantage of quasi-periodic properties of ECG signals to accurately detect pulses localization with an original algorithm based on the fast Fourier transform (FFT). Intensive simulations were achieved in terms of relative errors, coherence and accuracy for different levels of ECG interference. And the correlation coefficients computed from the paraspinal muscles EMG signals of 12 healthy participants were also used to evaluate the developed method. The results from simulation and real data demonstrate that the proposed method accurately detects pulses positions and efficiently removes the ECG from EMG signals, even when both signals are strongly overlapped, and greatly limits the deterioration of the EMG.
\end{abstract}

(c) 2017 Elsevier Ltd. All rights reserved.

\section{Introduction}

Non-invasive and inexpensive, surface electromyography (sEMG) is an effective method that has been widely adopted in clinical and research works to study topics related to muscular activities such as neuromuscular diseases. Surface EMG electrodes provide a non-stationary electrical signal representing the sum of subcutaneous motor unit action potentials generated during a muscular contraction. However, before any interpretations of the obtained signals, post-processing is needed. Indeed, sEMG electrodes are very sensitive elements, and many artifact sources can corrupt the recorded signal: movement artifacts, interferences from power lines, others devices, or others body parts. Artifacts cancellation is a key topic in biomedical signal processing.

When a muscular activity is recorded in the thoracic or trunk region, the sEMG signals are strongly contaminated by the heart

\footnotetext{
* Corresponding author.

E-mail address: francois.nougarou@uqtr.ca (F. Nougarou).
}

muscles electrical activity (ECG - electrocardiogram). In this case, it is difficult to separate the heart contribution from the muscular one, because their frequency spectra overlap: (i) from 10 to $500 \mathrm{~Hz}$ with most power between $20-200 \mathrm{~Hz}$ for EMG and (ii) from 0 to $100 \mathrm{~Hz}$ for ECG with a power failing after $35 \mathrm{~Hz}$ [1]. Cardiac artifacts clearly corrupt muscular responses. This corruption is more damaging if the signals to analyse are low muscular activities, as for example, it is the case in studies about the impacts of the spinal manipulation therapy on the human physiologic responses [2-4]. In those experimentations, sEMG electrodes recorded spine erector spinae muscles (close to thoracic vertebrae) obtained during spinal manipulation realized by a servo-controlled motor [2]. Those sEMG sensors responses are low-amplitude signals and are strongly contaminated by cardiac pulses, because of their position. To address this situation, the present paper attempts to propose an effective algorithm to remove the ECG, while maintaining the maximum information from targeted muscles.

Due to the biomedical engineering community interest, many methods to remove ECG from EMG signals have been proposed in the literature as exposed in [5,6]. Simple high-pass filters used in 
[1,7] do not represent an acceptable solution to reach this study's objective: EMG information is too much deteriorated. Based on an additional electrode, which records only ECG, the adaptive noise canceller (ANC) structure presents a powerful solution to remove ECG from EMG signal. This method has been used from respiratory and trapezius muscles in [8,9], both with RLS (Recursive Least Square) [10]. Authors in [11] propose a modified version of FBLMS (Fast Block Least Mean Squares) to adjust the filter coefficients of ANC, which returns better performance to cancel ECG than RLS. However, ANC performances are sensitive to synchronisation between the ECG contribution from both electrodes (sEMG and ECG reference electrodes) and are often weak in case of real signals. As the ANC method, the adaptive line enhancer (ALE) [12] is a structure based on adaptive filtering, which does not require a reference signal to remove ECG from EMG as in $[13,14]$. This technique allows to efficiently remove signal noise and to separate periodic or quasi-periodic narrowband components from a wideband Gaussian signal. Nevertheless, ALE cannot perform if the noise is not white Gaussian [13], and it can also remove periodic or quasi-periodic parts of desired EMG signal.

Independent Component Analysis (ICA) [15] and Singular Spectrum Analysis (SSA) [16], two methods of blind sources separation, have been also used to substrate ECG from sEMG electrodes. Based on several sEMG electrodes, some methods use ICA to first decompose EMG signals into statistically independent components. Then, the components containing ECG are discarded and remaining components are used to construct cleaned signals. In [17], ICA components corresponding to ECG were determined using the periodic characteristics of cardiac pulses (RR interval). This process was applied on trunk, spinal lumbar and diaphragm muscles in [17-21]. However, ECG and EMG contributions are not perfectly separated with ICA, and, when ECG components are totally removed, EMG substantial values are lost. This deterioration is minimized by authors in $[15,22]$. They use a high-pass filter at $30 \mathrm{~Hz}$ cut-off frequency on ECG components before reconstructing signals. Contrary to ICA, based on several sEMG electrodes, SSA method uses several delayed versions of a single signal to extract independent components [15]. A technique based on SSA to determine and cancel the ECG contribution of the signal was presented in [14,23,24]. It outperforms the ALE [14], but some errors occur when the periodic signal (ECG) is not narrowband or well defined [13]. In order to overcome this problem, a combination of SSA and ALE was suggested in [13] and improved in [25], in which the ALE structure selects adaptively the ECG component for wideband periodic signals disturbed by non-Gaussian noise. As classic ALE, this method can deteriorate EMG by removing its periodic components.

Widely applied in bio-signals processing such as events detection [26], classification [27] or artifacts cancellation, wavelet transform (WT) is a powerful time-frequency approach $[28,29]$. However, WT degrades EMG when it is directly used to remove ECG. Better performances are obtained when the ICA method is combined to WT in order to select and cancel ECG components as proposed in [30-32]. This structure has been designed with discrete and continuous wavelet transform in $[33,34]$.

Those techniques based on ICA and WT, like all other powerful methods exposed previously, apply their treatment along the whole signal, even during noiseless sections: they efficiently remove ECG, but greatly deteriorate the desired EMG. The preservation of the EMG contribution during the ECG cancellation is essential in this study. For this reason, the proposed method is based on a local cancellation approach. Contrary to other approaches, this method does not directly cancel the ECG; it first focuses on the ECG localization, before removing it only where pulses positions have been estimated (cancellation phase). The major contribution is the localization phase that is able to accurately locate cardiac pulses, even if the ECG and EMG amplitudes are close. First, the ECG localization realized by efficiently combining the discrete wavelet transform (DWT) and ICA to extract the ECG information from the sEMG electrodes. Then, using this extracted information, an original pulses detection based on fast Fourier transform (FFT) takes advantage of the quasi-periodic nature of the ECG to find the cardiac pulses positions with a strong accuracy. This step of the proposed method is essential to greatly remove ECG with limited EMG deteriorations. Indeed, as often wrongly assumed in literature, ICA does not perfectly separate ECG from sEMG; particularly in case of real data or when EMG and ECG contributions are closed.

The efficiency of the combination of DWT and ICA to first localize cardiac pluses and the impact of the two phases structure (localization and cancellation) to remove ECG from sEMG have been preliminary presented and evaluated in a conference paper [30]. In order to precisely describe the complete version of the proposed method (with addition of the quasi-periodic ECG pulses detection) and to realize an intensive evaluation, the present paper is organized as follows: the Section 1 describes the signals model used for simulation. Section 2 details the main parts of the proposed method for ECG cancellation: the ECG localization with extraction and detection of the cardiac signal, followed by its cancellation. A particular focus on the proposed algorithm of ECG pulses detection based on FFT is exposed in Section 3. The proposed method is then evaluated in Section 4. Intensive simulations have been achieved to determine the impact of each part of the proposed method in terms of relative errors (time-domain), coherence (frequency-domain) and accuracy of its pulses detection. Simulations were conducted for different levels of ECG interference on EMG; the results obtained by methods based on classic filtering and SSA are used for comparisons. Furthermore, in Section 5, the performances of proposed method are evaluated with real data obtained during the study of spinal manipulation effects [2-4]. Finally, some brief conclusions are drawn in Section 6.

\section{Signal model}

In the signal model, realized to evaluate ECG cancellation methods, $K$ fictive closed sEMG electrodes are considered, which record muscular activities contaminated by ECG from the same source. As defined in (1) and presented in Fig. $3 a, s_{k}[n]$ is the sum of the muscular contribution $s_{k}^{\mathrm{EMG}}[n]$ (information) and the ECG component $s_{k}^{\mathrm{ECG}}[n]$ (interference) of each electrode. The indexes $k=1,2$, $\ldots, K$ and $n=1,2, \ldots, N$ are respectively referred to the electrodes and samples, with $N$ the samples number and $F_{S}$ the sampling frequency.

$s_{k}[n]=s_{k}^{\mathrm{EMG}}[n]+s_{k}^{\mathrm{ECG}}[n]$

The interference levels are controlled with the following SIR (Signal to Interference Ratio) expression in $\mathrm{dB}$, noted $r_{k}^{\mathrm{SIR}}$ for a given sEMG electrode $k$ :

$r_{k}^{\mathrm{SIR}}=10 \log \left(P_{s_{k}} / P_{s_{k}^{\mathrm{ECG}}}\right)$

where $P_{s_{k}}$ is the power of the signal $s_{k}[n]$ recorded by the electrode $k$, and $P_{s_{k}}^{\mathrm{ECG}}$ the power of its ECG contribution.

The simulated EMG contribution $s_{k}^{\mathrm{EMG}}[n]$ of $s_{k}[n]$ are obtained from an autoregressive modelling described in [27]. For each electrode $k$, the spectral content obtained from a real EMG without ECG is first inserted in a white noise with a normal distribution. Then, the obtained signals are modulated with an envelope shape which represents repeated muscle contractions (see obtained EMG signals in Fig. 1a). The same envelope shape is used for all $K$ electrodes. Based on this process, all synthetized EMG responses are closed but different. 

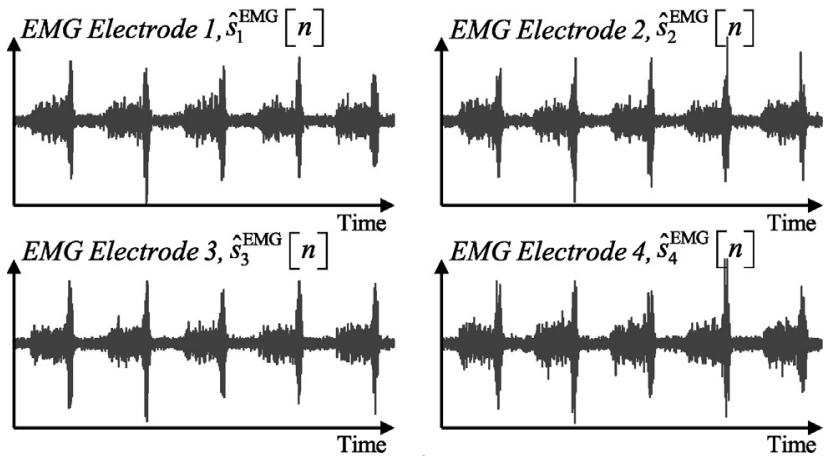

a)
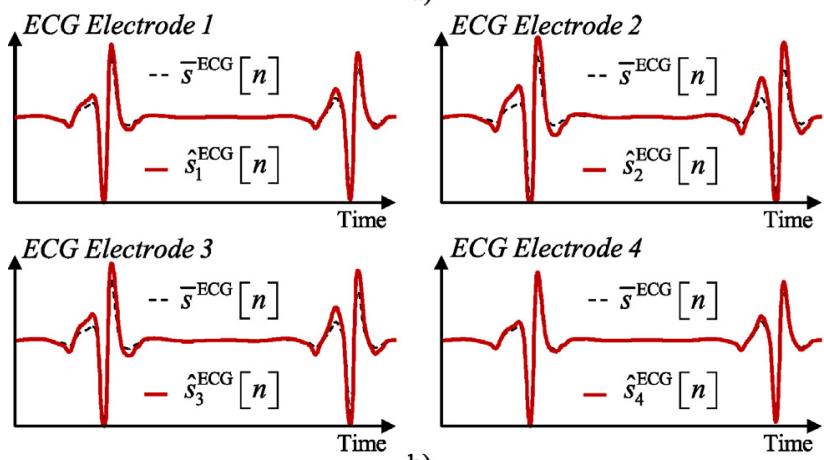

b)

Fig 1. a) Example of obtained EMG simulated signals with $K=4$ electrodes for $r^{S I R}=13 \mathrm{~dB}$. b) ECG simulated signals (focused on two pulses) obtained from (2) for $K$ electrodes in comparison with the original real ECG signal.

Even if the source of ECG artifacts is the same for all $K$ sEMG electrodes, all $s_{k}^{\mathrm{ECG}}[n]$ contributions are not exactly the same, due to the ECG signal propagation in tissues to each electrode. The $K$ signals $s_{k}^{\mathrm{ECG}}[n]$ are computed from the same real ECG signal, noted $\bar{s}_{k}^{\mathrm{ECG}}[n]$, according to expression (3).

$s_{k}^{\mathrm{ECG}}[n]=g\left(\bar{s}_{k}^{\mathrm{ECG}}[n]\right)=\gamma_{k}\left(\frac{1}{1+\exp \left(\lambda_{k}+\bar{s}_{k}^{\mathrm{ECG}}[n]\right)}-1\right)$

This expression induces a small non-linearity to generate a small deformation of $\bar{s}_{k}^{\mathrm{ECG}}[n]$ to obtain $s_{k}^{\mathrm{ECG}}[n][11] . g(\bullet)$ is a non-linear function, $\lambda_{k}$ sets the degree of non-linearity of the function and $\gamma_{k}$ controls the amplitude and the sign of the function output. $\lambda_{k}$ and $\gamma_{k}$ are randomly fixed in a limit range in order to induce significant, but small deformations between all $K$ electrodes and $\bar{s}_{k}^{\mathrm{ECG}}[n]$ as shown in Fig. 1 b.

\section{Proposed method of ECG cancellation}

The proposed method, presented in Fig. 2, seeks to minimize the deterioration of the EMG contribution during the ECG removal process. To do that, this method, which takes advantage of the use of several sEMG electrodes, is based on two main phases: ECG local- ization and ECG cancellation based on knowledge of cardiac pulses positions.

Details about main steps of the proposed method are described in the following paragraphs. The Fig. 3 illustrates results obtained from simulated data of 4 sEMG electrodes. In order to clarify the method description, it is important to make a difference between the terms "position" and "localization". The position of an ECG pulse represents one single value of sample (it can refer to the maximum amplitude of the pulse) and the localization of an ECG pulse corresponds to the area (a group of samples) where the overall pulse is located.

\subsection{ECG localization: DWT/ICA combination}

The ECG localization first extracts the ECG contribution by performing a combination of the DWT [28] and the ICA. As mentioned in [30], the DWT acts as frequency/shape filtering configured to extract ECG component of each recorded signal. Indeed, based on a given "mother wavelet" $\psi[n]$, DWT decomposes all inputs $s_{k}[n]$ in several time-domain sub-signals, noted $d_{k}^{j}[n]$, of different frequencies ranges, named levels and referred as $j=1,2, \ldots, J$; with $J$ the total number of levels. The frequency/shape filtering is then performed by selecting pertinent levels $j$. Because the frequency range of main ECG components is between $20 \mathrm{~Hz}$ and $50 \mathrm{~Hz}$, wavelet levels of $j=[4,5,6]$ are used according to $F_{S}=1000 \mathrm{~Hz}$. Considering $\psi[n]$ with a shape close to cardiac pulse, the $k^{\text {th }}$ frequency/shape filtering result $x_{k}[n]$ is a sum of time-series $d_{k}^{j}[n]$ obtained from $s_{k}[n]$ for selecting levels (4). Examples of obtained signals $x_{k}[n]$ are exposed in Fig. 3b.

$x_{k}[n]=\sum_{j=4}^{6} d_{k}^{j}[n]$

As shown in Fig. $3 \mathrm{a}$ and b, the signals $x_{k}[n]$ contained essentially ECG information but also some residues of the EMG contribution which can alter the ECG localization. ICA is applied on the DWT frequency/shape filtering results of all sEMG sensors to improve extraction of ECG information. Indeed, as exposed in [22] and [30], the ICA technique permits to decompose some inputs into statistically independent components from each other. Based on $x_{k}[n]$, the ICA method determines some independent sources $y_{k}[n]$ for all $k$. As presented in Fig. 3c, the ICA allows regrouping the common information from the signals $x_{k}[n]$. The 3rd ICA component of Fig. $3 c$ corresponds to ECG independent component which has been clearly separated from its residues.

\subsection{ECG localization: quasi-periodic ECG pulses detection}

Based on the ICA independent component $y_{k}[n]$, the "Quasiperiodic pulses detection" block in Fig. 2 applies a detection method taking advantage of the quasi-periodic nature of the ECG signal. It returns an estimation of the cardiac pulses localization, noted $\hat{\ell}[n]$ (5). As shown in Fig. $3 d, \hat{\ell}[n]$ does not represent a single sample per pulse, but an estimated area where the pulses are located. Details

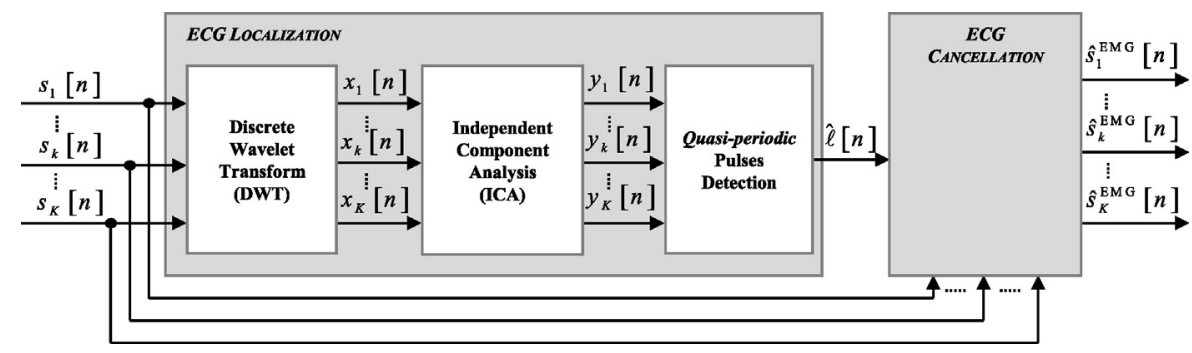

Fig. 2. Description of the proposed ECG cancellation method for $k=1,2, \ldots$, Kand $n=1,2, \ldots, N$. 


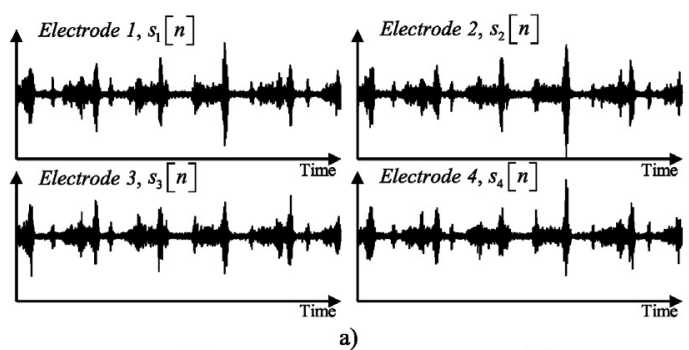

a)

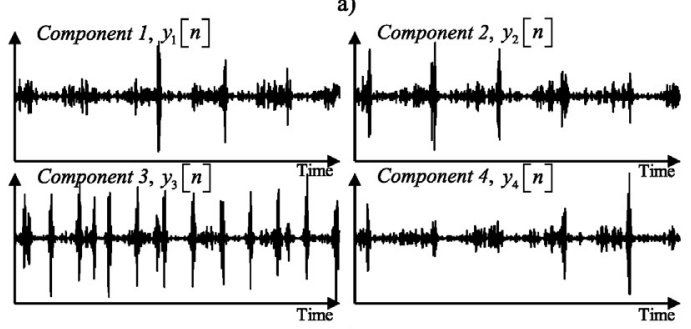

c)
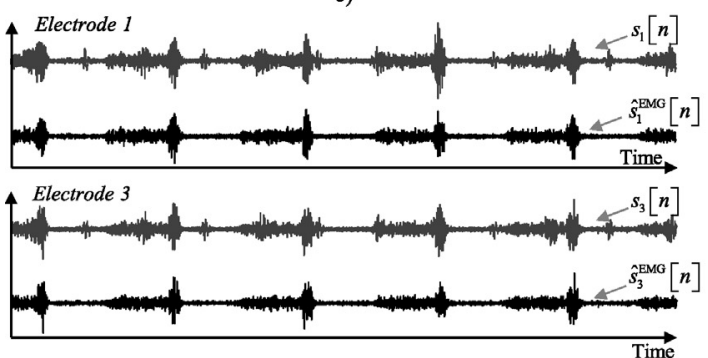

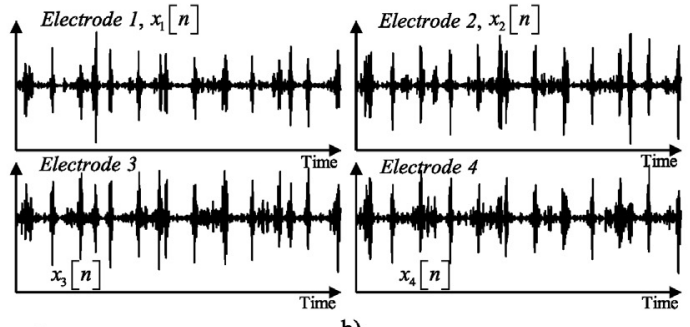

b)

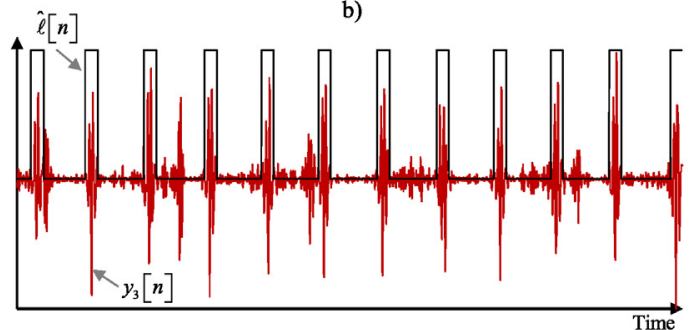

d)
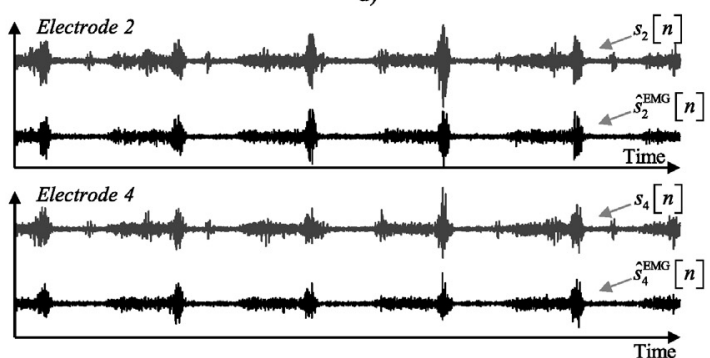

e)

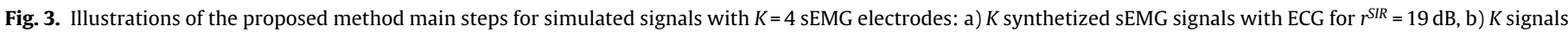

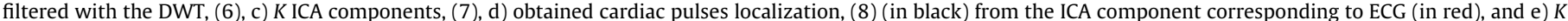

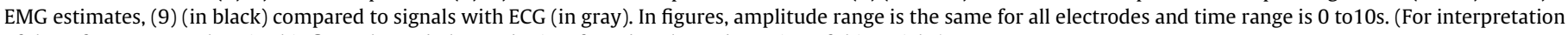
of the references to colour in this figure legend, the reader is referred to the web version of this article.)

on the quasi-periodic ECG pulses detection process are exposed in the Section 4.

$\hat{\ell}[n]=\left\{\begin{array}{cc}1, & \text { if ECG pulses are present } \\ 0, & \text { else. }\end{array}\right.$

\subsection{ECG cancellation}

Many techniques are available to cancel the ECG contribution. In the proposed method, $K$ signals $\sigma_{k}[n]$, which represent the ECG, are obtained from all signals $s_{k}[n]$ by applying a simple 4th order Butterworth low-pass filter of $50 \mathrm{~Hz}$. As exposed in (6), the $k^{\text {th }}$ EMG contribution signal $\hat{s}_{k}^{\mathrm{EMG}}[n]$ (Fig. 3e) is computed by subtracting the signal $s_{k}[n]$ of the $k^{\text {th }}$ SEMG sensor (Fig. 3a) with the product the signal $\sigma_{k}[n]$ and the vector of ECG localization $\hat{\ell}[n]$. For all electrodes $k$, the ECG component is subtracted only where pulses have been detected to limit the deterioration of EMG information.

$\hat{s}_{k}^{\mathrm{EMG}}[n]=s_{k}[n]-\sigma_{k}[n] \hat{\ell}[n]$

\section{Quasi-periodic ECG pulses detection method}

Even if the combination of wavelet transform and ICA is efficient to extract the ECG contribution from a set of sEMG recorded signals, the automatic localization of ECG pulses remains a non-trivial problem: some mistakes on the ECG position can easily appear, some artifact events being considered as ECG pulses. As mentioned in Section 3.2, the proposed ECG pulse detection method is based on the quasi-periodic nature of the ECG signal to estimate its pulses localization. Instead of a commonly used amplitude threshold, the proposed method uses a rule of periodicity to determine ECG pulses positions to create the ECG pulses localization signal $\hat{\ell}[n]$ (5). To do that, main events positions and periodic events positions of the extracted ECG contribution are compared to estimate $\hat{\ell}[n]$. The main blocks functions of this procedure are presented in Fig. 4 and described in the following paragraphs.

\subsection{ECG signal \& periodic frequency selection}

The first step of the proposed pulses localization method consists in automatically finding $y_{s}[n]$, the ICA independence source corresponding to the ECG component from $y_{k}[n]$, and its fundamental frequency, named the frequency of periodicity $F_{p}$. The ECG component is the one which presents a clear periodicity. The periodicity of a signal can be observed in the frequency domain, not directly by applying the FFT on signals $y_{k}[n]$ for all $K$, but on their rectified versions $y_{k}^{\text {rect }}[n]$, as depicted in (7) and presented in Fig. 5a:

$$
Y_{k}[f]=\mathrm{fft}\left(y_{k}^{\mathrm{rect}}[n]\right)
$$

with

$y_{k}^{\mathrm{rect}}[n]=\left|y_{k}[n]\right| / \max \left(y_{k}[n] \mid n=1,2, \ldots, N\right)$

where $\operatorname{fft}(\bullet)$ is the fast Fourier transform function, $|\bullet|$ is the absolute value function and $Y_{k}[f]$, illustrated in Fig. 5.b for all $K$, is the magnitude of the frequency representation of the $k^{\text {th }}$ ICA rectified component $y_{k}^{\text {rect }}[n]$ with $f=1,2, \ldots, N$. Note that rectified signals have also been normalized by their maximum value, with the function $\max (\bullet)$, to allow a fair comparison between the obtained frequency responses. In Fig. 5.b, the magnitude corresponding to the ECG signal presents the highest fundamental and harmonics peaks, which attest the presence of periodicity. The rectified transformation stands out the periodicity of signals, since it reveals 


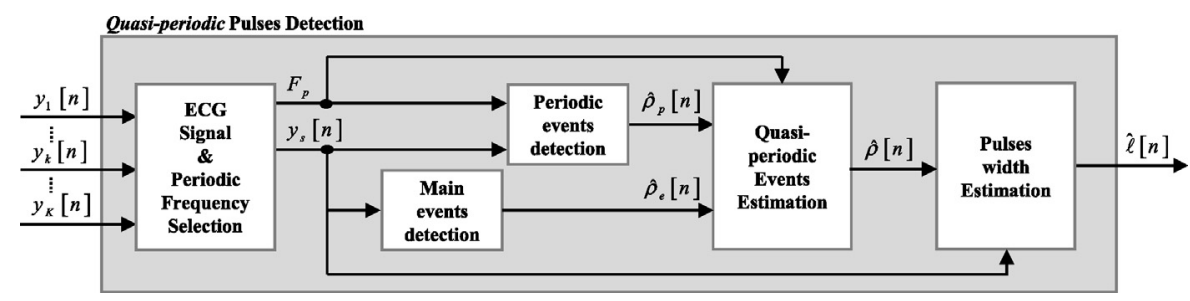

Fig. 4. Description of the proposed quasi-periodic pulses detection method for and.
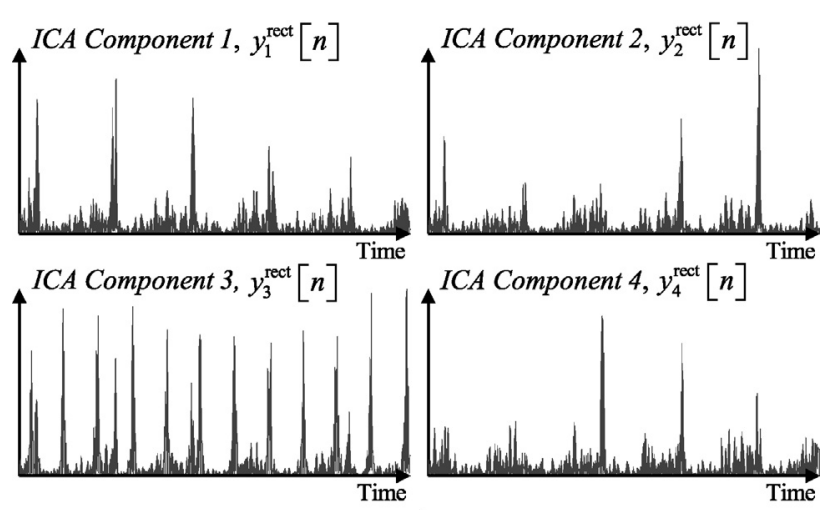

a)

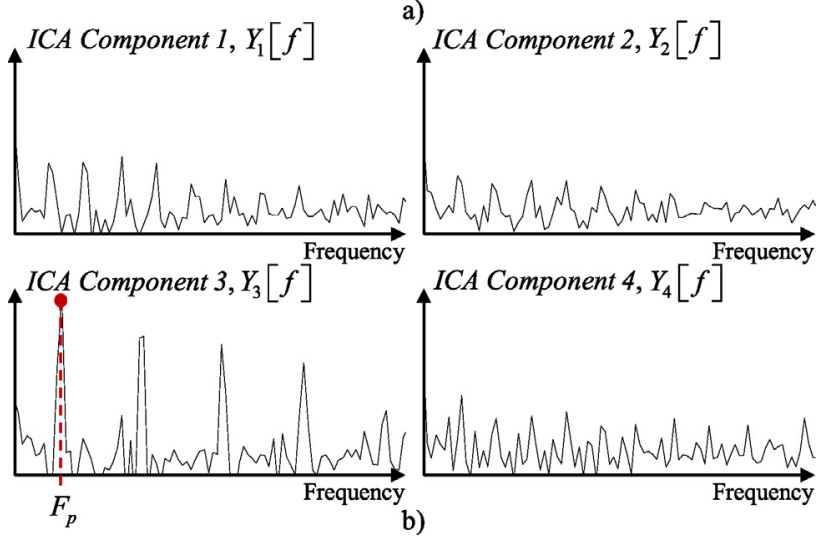

Fig. 5. Illustration of the "ECG signal \& periodic frequency selection" block with a) $K$ rectified components from ICA process and b) magnitudes of frequency representations of signals presented in a). The frequency representation of the ICA component corresponding to ECG contribution (the 3th one) presents a remarkable fundamental frequency named frequency of periodicity and noted $F_{p}$. In figures, amplitude range is the same for all electrodes, time range is 0 to $10 \mathrm{~s}$ and frequency range is $0,5 \mathrm{~Hz}$ to $6 \mathrm{~Hz}$.

their global behavior. In this paper, the considered frequency range of interest of the ECG signal is between $1 \mathrm{~Hz}$ and $2 \mathrm{Hhz}$, which correspond to 60 and 120 pulses per minute. Consequently, the index $k$ of $Y_{k}[f]$ which presents the highest peak of frequency (fundamental frequency) between $1 \mathrm{~Hz}$ and $2 \mathrm{~Hz}$, corresponds to the ECG signals and is noted $k_{\mathrm{ECG}}$. The rectified ECG component is noted $y_{s}[n]=y_{k_{\mathrm{ECG}}}^{\text {rect }}[n]$ and $F_{p}$ is the fundamental frequency of $Y_{k_{\mathrm{ECG}}}[f]$ (see Fig. 5.b).

\subsection{Main events detection}

The objective of this block presented in Fig. 4 consists in finding the main events positions $\hat{\rho}_{e}[n]$ from the rectified ECG component $y_{s}[n]$. To do that, a sliding-windows method, where variance is computed in each window, is applied to differentiate important events of $y_{s}[n]$ from no-pertinent one. The resulting signal is then smoothed with a low pass filter and referred as $\bar{y}_{s}[n]$ (see in gray in Fig. $6 \mathrm{~d}) . \bar{y}_{s}[n]$ allows standing out main peaks which are detected with a peaks detection method to obtain $\hat{\rho}_{e}[n]$ (8). As shown in Fig. 6.d (back dotted lines), $\hat{\rho}_{e}[n]$ contains the precise positions of ECG pulses, but also artifact positions.

$\hat{\rho}_{e}[n]=\left\{\begin{array}{cc}1, & \text { if position of } \bar{y}_{s}[n] \text { main event } \\ 0, & \text { else. }\end{array}\right.$

\subsection{Periodic events detection}

In order to estimate quasi-pure periodic events positions of $y_{s}[n]$, noted $\hat{\rho}_{p}[n](10)$, it is first necessary to construct a signal representing main periodic events of $y_{s}[n], y_{s}^{p}[n]$. The idea behind that is to apply on $y_{s}[n]$ a very selected band-pass filter centered on the frequency of periodicity, $F_{p}$. However, because strong amplitudes influence the filtering result, $y_{s}[n]$ is transformed before the filtering in order to attenuate the effect of important events. This transformation, based on logarithm function $\log (\bullet)$ called Shannon entropy in [35], is described in Eq. (9). Compared to $y_{s}[n]$ in Fig. 6a, the resulting signal $y_{s}^{\log }[n]$ which appeared in Fig. $6 \mathrm{~b}$ shows that all peaks have now the same amplitude level and that the influence of extreme peaks is cancelled.

$y_{s}^{\log }[n]=-\left|y_{s}[n]\right| \log \left(\left|y_{s}[n]\right|\right)$

$y_{s}^{\log }[n]$ is then filtered with a very selected band-pass filter centered on the frequency of periodicity, $F_{p}$, to obtain $y_{s}^{p}[n]$ Fig. $6 \mathrm{c}$ in gray. Periodic peaks positions of $y_{s}^{p}[n]$ are then detected and stored in $\hat{\rho}_{p}[n]$ (black lines in Fig. 6c):

$\hat{\rho}_{p}[n]=\left\{\begin{array}{cc}1, & \text { if position of } y_{s}^{p}[n] \text { main event } \\ 0, & \text { else. }\end{array}\right.$

Contrary to $\hat{\rho}_{e}[n]$, in $\hat{\rho}_{p}[n]$, the number of ECG pulses positions is equal to the number of pulses to detect, but due to the strict periodic rule, those positions are not precise as illustrated in Fig. 6c and $\mathrm{d}$.

\subsection{Quasi-periodic events estimation}

In the "Quasi-periodic events estimation" block, final estimated pulses positions $\hat{\rho}[n]$ are obtained by using an algorithm, described in Table 1, which determines good positions of $\hat{\rho}_{e}[n]$ (Fig. 6d) in function of $\hat{\rho}_{p}[n]$ (Fig. 6c). $v_{e}[u]$ contains the samples values corresponding to $\hat{\rho}_{e}[n]=1$ and $v_{p}[w]$ the samples corresponding to $\hat{\rho}_{p}[n]=1$, where $u=1,2, \ldots, N_{e}$ and $w=1,2, \ldots, N_{p} . N_{e}$ and $N_{p}$ are respectively the number of main events and periodic events. For a given $w$, the vector of distances, $\boldsymbol{\delta}_{w}$, of position $v_{p}[w]$ from the periodic positions $v_{e}[u]$ for all $u$ is computed in lines $2-4$ of Table 1 where $\boldsymbol{\delta}_{w}=\left[\delta_{w}[1], \ldots \delta_{w}[u], \ldots \delta_{w}\left[N_{e}\right]\right]^{T}$. For a given position $w$, $\delta_{w}^{\min }$ is the minimum distance of $\boldsymbol{\delta}_{w}$ for all $u$ and $u_{w}^{\min }$ this minimum value index (see line 5 in Table 1 ).

Considering $\hat{\boldsymbol{\rho}}$ a null vector with $\operatorname{dim}(\hat{\boldsymbol{\rho}})=N_{p} \times 1$ at the beginning of the algorithm, the final estimated positions are determined for all index $w$ in function of $\delta_{w}^{\mathrm{min}}$ as described in lines $6-10$ of Table 1 . For a given $w$, if the minimum $\delta_{w}^{\min }$ distance is inferior 


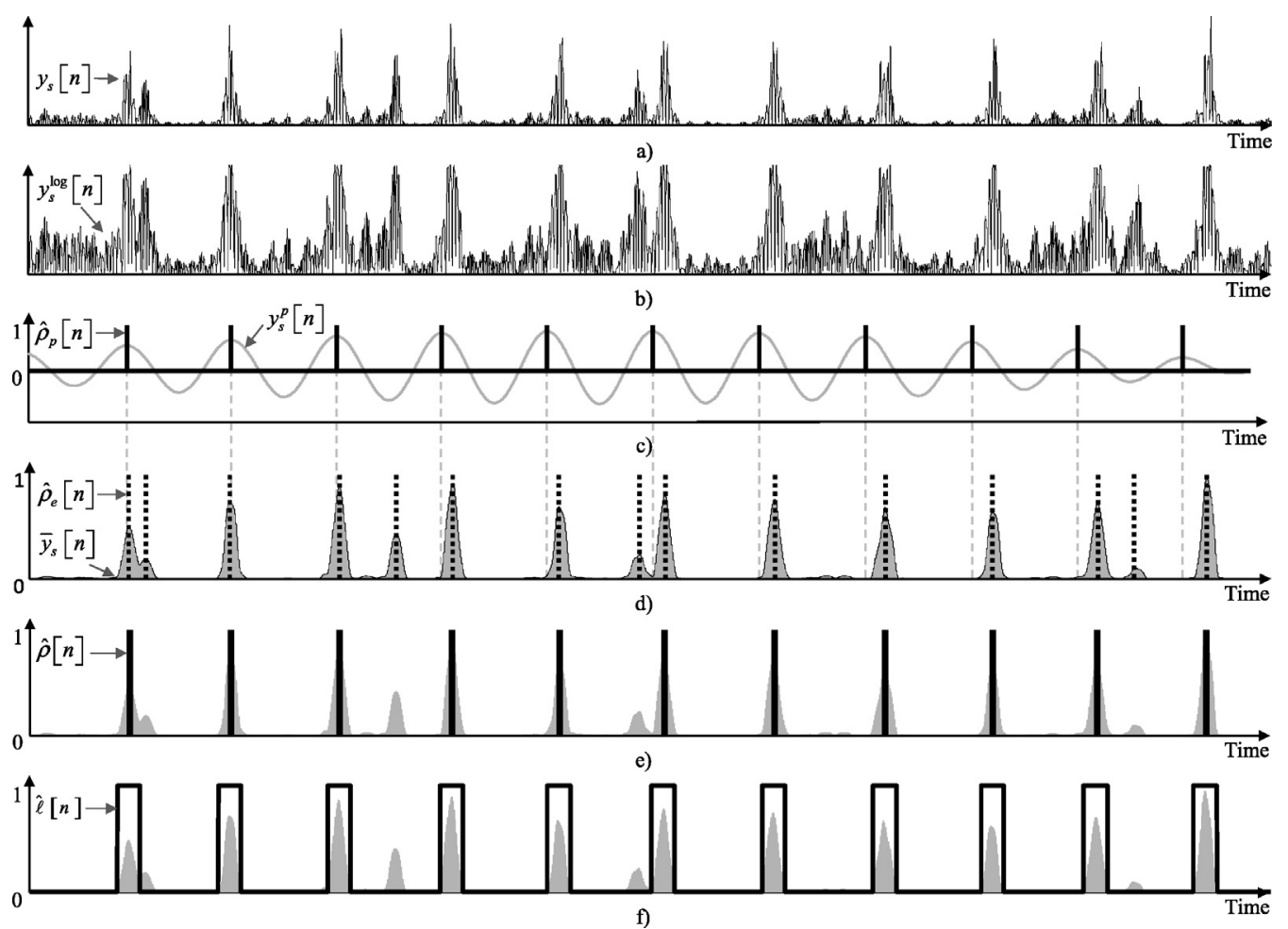

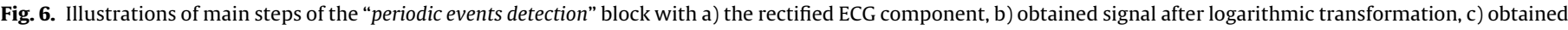

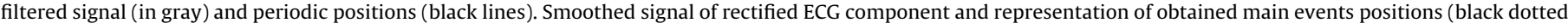

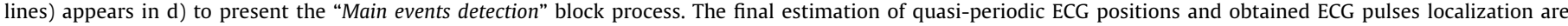
respectively illustrated in e) and f) (black lines) with the smoothed signal of rectified ECG component (in gray). Time range of figures is 0 to10s.

\section{Table 1}

Detection algorithm of quasi-periodic positions of the ECG signal, $\hat{\rho}[n]$ based on main events positions $\hat{\rho}_{e}[n]$ and from periodic events positions $\hat{\rho}_{p}[n]$ for $n=1,2, \ldots, N$.

\footnotetext{
Inputs \& Initialisation

$v_{p}[w]$ : vector of samples corresponding to $\rho_{p}[n]=1$

$v_{e}[w]$ : vector of samples corresponding to $\rho_{e}[n]=1$

$F_{s} \& F_{p}$ : sampling frequency $\&$ frequency of periodicity

$\hat{\boldsymbol{\rho}}=[0,0, \ldots, 0]^{T}$ with $\operatorname{dim}(\hat{\boldsymbol{\rho}})=N_{p} \times 1$
}

Process

\begin{tabular}{ll}
\hline 1 & for $w=1,2, \ldots, N_{p}$ \\
2 & for $u=1,2, \ldots, N_{e}$ \\
3 & $\delta_{w}[u]=\left|v_{p}[w]-v_{e}[u]\right|$ \\
4 & end \\
5 & $\left\{\delta_{w}^{\min }, u_{w}^{\min }\right\}=\min \left(\delta_{w}[u] \mid u=1,2, \ldots, N_{e}\right)$ \\
6 & if $\delta_{w}^{\min }>F_{s} / 2 F_{p}$ \\
7 & $\hat{\rho}\left[v_{p}[w]\right]=1$ \\
8 & else \\
9 & $\hat{\rho}\left[v_{p}\left[u_{w}^{\min }\right]\right]=1$ \\
10 & end \\
11 & end \\
\hline
\end{tabular}

or equal to half periodic sample, $F_{S} / 2 F_{p}$, the main events position of this minimum distance $v_{e}\left[u_{w}^{\min }\right]$ is considered 'good'. If the minimum distance is superior to $F_{s} / 2 F_{p}$, the main events position of the minimum distance is too far from the periodicity and is not considered 'good'. In this case, the periodic position of the index $w, v_{p}[w]$, is considered.

\subsection{Pulses width estimation}

In order to construct the signal of estimated ECG localization $\hat{l}[n]$ (see Fig. 6f), it is first necessary to estimate the width of the ECG pulses. This step is based on the extracted ECG contribution $y_{s}[n]$ and the estimated ECG positions $\hat{\rho}[n]$. As realized in the "Main events detection" block, $y_{s}[n]$ is modified to obtain a smoothed version noted $\bar{y}_{s}[n]$. And the vector of samples corresponding to $\hat{\rho}[n]=1$, referred as $v[w]$ for $w=1,2, \ldots, N_{p}$, is also considered. For each estimated ECG pulse $w$, a threshold $\tau_{w}$ is computed from the peak value of the $w^{\text {th }}$ pulse $\bar{y}_{s}[v[w]], \tau_{w}=\alpha \bar{y}_{s}[v[w]]$, where $\alpha$ is a chosen percentage (in this project $\alpha=0.5 \%$ ). Then $\tau_{w}$ is applied at left and right side of $v[w]$, by keeping values superior to $\tau_{w}$ to estimate the width $\Delta[w]$ of this $w^{\text {th }}$ pulse. In order to reject the extreme values of determined widths, the median value of width, $\Delta^{\text {med }}=$ median $\left(\Delta[w] \mid w=1,2, \ldots, N_{p}\right)$ is considered. The signal of ECG pulses localization $\hat{\ell}[n]$ is then obtained as a convolution of ECG pulses positions $\hat{\rho}[n]$ with a rectangular window of size $\Delta^{\text {med }}$.

\section{Performance evaluations}

\subsection{Simulation parameters, methods and evaluation criteria}

In order to evaluate the proposed method, $K=4$ sEMG electrodes were considered. According to (1) and (2), simulations were conducted for 9 levels of ECG contamination: SIR $=[1,3,5,7,10,15,20,25,30] \mathrm{dB}$ where the smaller SIR value represents a stronger impact of ECG on the EMG contribution. For each SIR value, methods of ECG cancellation were performed $N_{i}=300$ times. As exposed in Section 2, based on random process, signals $s_{k}[n]$ for all $k$, all SIR values and all simulation repetitions are different, and their duration is equal to $10 \mathrm{~s}(N=10000)$.

The proposed method, referred as $M 3$, uses in its DWT filtering process a mother wavelets "db3" which has a shape close to a cardiac pulse and wavelet levels fixed in Section 3.1. For the sake of comparison, $M 3$ is evaluated through simulation iterations with other techniques of ECG removal listed in Table 2. $M 0$ shows results obtained when no ECG remove method is used, in order to observe 
Table 2

Simulated methods to cancel ECG and their description.

\begin{tabular}{ll}
\hline Methods Reference & Descriptions \\
\hline M0 & $\begin{array}{l}\text { No method employed } \\
\text { Method based on a 4th order Butterworth high-pass at } \\
\text { cut-off frequency of } 50 \mathrm{~Hz}\end{array}$ \\
M2 & $\begin{array}{l}\text { Method based on singular spectrum analysis (SSA) } \\
\text { M3 }\end{array}$ \\
Proposed method in Fig. 2, with DWT, ICA and \\
pseudo-periodic pulses detection \\
Method based on $M 3$ but without pseudo-periodic pulses \\
M5 & $\begin{array}{l}\text { detection } \\
\text { pulses detection }\end{array}$ \\
M6 & $\begin{array}{l}\text { Method based on } M 3 \text { with more accurate cut-off frequency } \\
\text { values in its high-pass filter }\end{array}$ \\
\hline
\end{tabular}

the levels of degradation generated by the ECG on the EMG signals for a given SIR value. Methods $M 1$ and M2, respectively based on classic filtering [1] and singular spectrum analysis (SSA) [16], directly attempt to remove the ECG from the sEMG without cardiac pulses localization. $M 2$ has been chosen because SSA presents an excellent capacity to extract a periodic component (ECG) from a given signal (EMG) [14] and [23]. Methods M4 to M6 represent particular case of M3.M4 and M5 allow observing the effect of quasiperiodic pulses detection and the combination of DWT and ICA, respectively. $M 4$ is the method presented in the conference paper [30]. M6 differs from $M 3$ in chosen cut-off frequency values $f_{c}$ of high-pass filter used during the ECG cancellation. M6 takes into account the amplitude level of the ECG compared to EMG. Three levels of the ECG to EMG amplitude ratio are considered: (i) if the ratio is high $f_{c}=50 \mathrm{~Hz}$, (ii) if it is medium $f_{c}=45 \mathrm{~Hz}$ and (iii) if it is low $f_{c}=40 \mathrm{~Hz} M 6$ allows analyzing the impact of a more accurate technique in the "ECG cancellation" block of Fig. 2 .

The relative error $E_{k}$ has been chosen to evaluate time-domain similarity between the EMG contribution and its estimate, $s_{k}^{\mathrm{EMG}}[n]$ and $\hat{s}_{k}^{\mathrm{EMG}}[n]$. Smaller relative error values reflect greater denoising performances. $E_{k}$ is computed as follow for each electrode $k$, method, and simulation iteration:

$E_{k}=\sum_{n=1}^{N}\left(s_{k}^{\mathrm{EMG}}[n]-\hat{s}_{k}^{\mathrm{EMG}}[n]\right)^{2} / \sum_{n=1}^{N}\left(s_{k}^{\mathrm{EMG}}[n]\right)^{2}$

In addition, a frequency-domain criterion is provided by the mean coherence [9], noted $\bar{C}_{k}(12)$ for all electrodes and methods at each iteration. In order to focus on the range of frequency contaminated by the ECG, the mean coherence is computed for frequencies between $10 \mathrm{~Hz}$ and $100 \mathrm{~Hz}$. Good denoising performance results in coherence values closed to 1 .

$\bar{C}_{k}=\operatorname{mean}\left(P_{s_{k}^{\mathrm{EMG}} \hat{s}_{k}^{\mathrm{EMG}}}[f]^{2} / P_{s_{k}^{\mathrm{EMG}} s_{k}^{\mathrm{EMG}}}[f] P_{\hat{s}_{k}^{\mathrm{EMG}} \hat{s}_{k}^{\mathrm{EMG}}}[f]\right)$

where $P_{s_{k}^{\mathrm{EMG}} \hat{s}_{k}^{\mathrm{EMG}}}[f], P_{s_{k}^{\mathrm{EMG}} s_{k}^{\mathrm{EMG}}}[f]$ and $P_{\hat{s}_{k}^{\mathrm{EMG}} \hat{s}_{k}^{\mathrm{EMG}}}[f]$ are cross- and auto-spectral densities of $s_{k}^{\mathrm{EMG}}[n]$ and $\hat{s}_{k}^{\mathrm{EMG}}[n]$, with $10 \mathrm{~Hz} \leq f$ $\leq 100 \mathrm{~Hz}$ as the frequency sample.

In order to evaluate the contribution of the quasi-periodic ECG pulses detection method detailed in Section 3, two others criteria have been computed for methods M3 to M6 at each iteration: (i) errors between the number of ECG pulses detected by the methods compared to the real number of ECG pulses, $\varepsilon_{p}$, and (ii) the mean distance between all estimated pulses positions and their closer true pulses positions, $\Delta_{p}$. A method of pulses detection is efficient of it is able to find the right number of pulses with a close distance.

For all four criteria, means has been computed along all iterations of simulation and all electrodes $\left(N_{i} \times K=300 \times 4\right.$ values per mean) for a given SIR value. The obtained criteria means in function of the SIR values for all methods are presented in Fig. 7 for $E_{k}$ (11) and $\bar{C}_{k}(12)$, and in Table 3 for mean number of pulses error $\varepsilon_{p}$, and mean distance error $\Delta_{p}$.
Table 3

Obtained results of the mean number of pulses error and mean distance error in function of SIR values for M3, M4 and M5.

\begin{tabular}{|c|c|c|c|c|c|c|}
\hline \multirow[t]{2}{*}{$\operatorname{SIR}(\mathrm{dB})$} & \multicolumn{3}{|c|}{ Mean number of pulses error, $\varepsilon_{p}$} & \multicolumn{3}{|c|}{ Mean distance error $(\mathrm{ms}), \Delta_{p}$} \\
\hline & M3 & M4 & M5 & M3 & M4 & M5 \\
\hline 1 & 0.00 & 0.00 & 0.03 & 3.48 & 3.48 & 4.14 \\
\hline 3 & 0.00 & 0.02 & 0.99 & 3.34 & 3.69 & 24.3 \\
\hline 5 & 0.00 & 0.13 & 1.87 & 3.44 & 6.62 & 42.1 \\
\hline 7 & 0.00 & 0.46 & 2.80 & 3.41 & 14.23 & 60.7 \\
\hline 10 & 0.00 & 1.02 & 3.69 & 3.46 & 25.51 & 78.8 \\
\hline 15 & 0.01 & 1.98 & 4.74 & 3.90 & 47.03 & 101 \\
\hline 20 & 0.00 & 2.97 & 5.87 & 5.14 & 64.85 & 118 \\
\hline 25 & 0.02 & 3.76 & 6.54 & 8.62 & 80.2 & 129 \\
\hline 30 & 0.16 & 4.61 & 6.66 & 19.3 & 101 & 142 \\
\hline
\end{tabular}

\subsection{Evaluation results of simulations}

The analysis of each method requires the information provided by all criteria calculated during the simulation. For this reason, the methods will be analyzed one by one from the results presented in Fig. $7 \mathrm{a}$ and $\mathrm{b}$, and Table 3 (if applicable) starting with methods $M 0$, M1 and M2:

- M0 results in Fig. 7 show the damage caused by the ECG on EMG for time-domain (Fig. 7.a) and frequency-domain (Fig. 7b) in function of the level of SIR. In those simulations, the ECG clearly interferes with the EMG signal.

- With its high-pass filter applied to the entire signal, M1 eliminates not only the ECG, but also the EMG. In Fig. 7a, the relative error of $M 1$ is higher than other methods. And observing the frequency domain in Fig. 7b, M1 deteriorates the EMG signal more than the addition of the ECG (MO).

- M2 fails to finely separate ECG component of EMG: its result in the frequency-domain cannot do better than MO. As the ECG signal, the synthetized EMG part of all electrodes (Fig. 1a) also present a periodicity, with repetitive muscular contractions. This fact disturbs the SSA method, which try to find periodic independent components of a given signal.

Below is a thorough analysis of the proposed method, M3. Methods M4 and M5, which are incomplete versions of $M 3$ are used here to understand the impact of various parts of $M 3$.

- As observed in Fig. 7a, M3 allows to clearly obtain smaller relative errors than $M 0, M 1$ and $M 2$, whatever the applied ECG level (SIR). Fig. 7b shows that $M 3$ also improves the frequency content of the degraded signal: for all SIR, the obtained frequency content is closer to the one of the signal without deterioration. M3 efficiently cancels the cardiac pulses from the EMG signal. The M3 results compared to M1 and M2 demonstrate the value of its two-steps strategy to remove ECG: localization and cancellation.

- Results in Table 3 show that the detection of the cardiac pulsation used in $M 3$ is very powerful. Whatever the ECG deterioration level, the error of detected pulses, $\varepsilon_{p}$, is less than 0.16 pulses (for SIR $=30 \mathrm{~dB}$ ). Furthermore, the positions of the detected pulses in $M 3$ are close to the positions of added pulses to EMG: maximum average distance between the detected and added pulses is $\Delta_{p}=19.3$ samples ( $\operatorname{SIR}=30 \mathrm{~dB}$ ), which is equivalent to $19.3 \mathrm{~ms}$. Even if the effect of the heartbeat is small compared to the EMG, M3 allows to accurately detect the ECG pulses.

- Contrary to M3, M4 does not take advantage of the periodic nature of the ECG signal in their pulses detection step. As shown in Fig. 7a, $M 4$ provides good relative error results: less than $M 0$, regardless of the interferences level. However, in Fig. 7b, M4 improves the frequency content of the interfered signal (greater than $M 0$ ) only until SIR $=20 \mathrm{~dB}$. Comparing $M 4$ to $M 3$, results are equal 


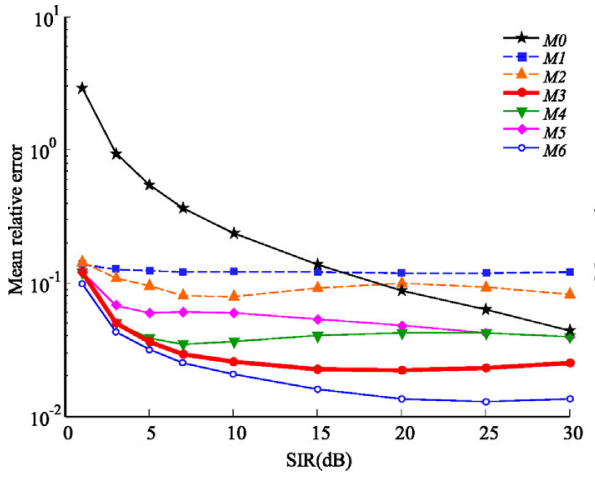

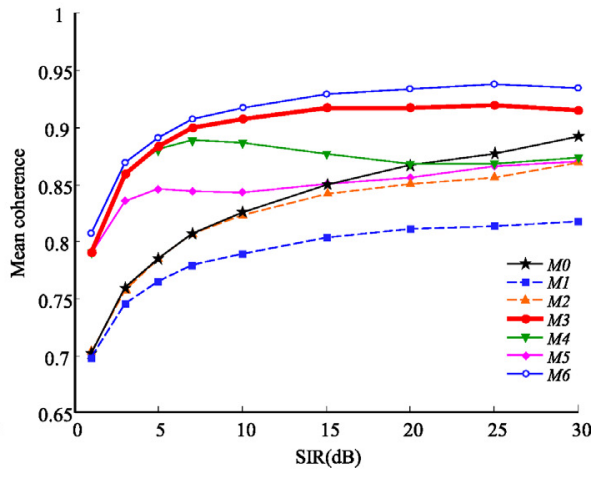

b)

Fig. 7. Obtained results for all ECG remove methods of (a) mean relative and (b) mean coherence in function of SIR values in dB.

until $7 \mathrm{~dB}$, but $M 4$ results deteriorate after this SIR level. The quasi-periodic pulses detection of Section 3 improves the ECG cancellation results of $M 3$. However, M3 and M4 return better results to remove ECG from EMG than methods based on ICA (as method proposed in [17]) which reject the entire ICA components corresponding to ECG. Indeed, contrary to those methods, $M 3$ and M4, with their two main phases preserve noiseless parts of the signal.

- The comparison of M4 and M5 allows evaluating the combination of DWT and ICA: M4 uses this combination [30], while M5 only uses DWT to extract the ECG component from the EMG signal prior to detection pulses (Fig. 2). Without ICA, M5 returns worse results in terms of relative error than $M 4$. The interest of the combination especially appears in Fig. 7b where the frequency content obtained with M4 is clearly closer to the frequency content of the signal without interference than M5. Furthermore, Table 3 shows that M5 has more errors in the detected pulses number and in accuracy than $M 4$ for all SIR levels.

- In Table 3, $\varepsilon_{p}$ and $\Delta_{p}$ increase for M4 and M5 when the level of interferences decreases. Indeed, it is more difficult to detect heartbeats when their influence is weak. However the error on the number of pulses remains almost constant and close to 0 for M3. Furthermore, the worst precision error obtained for $M 3$ at $\mathrm{SIR}=30 \mathrm{~dB}$ is achieved approximately by $M 4$ at $\mathrm{SIR}=10 \mathrm{~dB}$ and $M 5$ at SIR $=3 \mathrm{~dB}$. The combination of DWT, ICA and the detection based on the quasi-periodic signal events used in M3 efficiently localizes the ECG pulses, even when they are small compared to EMG.

Finally, once the ECG pulses are localized, several types of cancellation methods can be used. Methods M3 and M6 are identical except for their "ECG cancellation" block content. M6, exposed in Section 4.1, minimizes damage to the EMG signal when the impact of the ECG is lower. Regardless of the SIR values, relative errors and mean coherences are improved with M6 compared to M3. The proposed method, when the pulses are correctly localized and the cancellation method is well designed, can efficiently remove the ECG pulses from the EMG signal, even when they are more difficult to detect.

\subsection{Experimental validation on real subjects}

Some experimentations are conducted at the GRAN laboratory (chiropractic department, University of Quebec at Trois-Rivières, Qc, CA) to study fundamental aspects the spinal manipulation therapy (SMT) $[3,4]$. The manipulation was performed by a servocontrolled motor [2] on the 7th vertebrae thoracic (T7) (see an example of applied force curve in Fig. 8 first graph). Bipolar muscular responses were recorded around the impact with four Delsys
Surface EMG electrodes (Model DE2.1, Delsys Inc., Boston, MA, USA). Those sensors were positioned on each side of the spine over the thoracic spine erector spinae muscles: two sEMG sensors at $2 \mathrm{~cm}$ from the 6th vertebrae thoracic (T6) and two others from the 8th vertebrae thoracic (T8). Data were recorded with $F_{S}=1000 \mathrm{~Hz}$. Before applying the ECG cancellation methods, a $10-500 \mathrm{~Hz}$ bandpass 4th order Butterworth filter was applied in order to only consider the EMG information [1]. The power line interference was cancelled with notch filters at $60 \mathrm{~Hz}$ and its harmonics.

The behavior of the proposed method (M3) with real data is observed in Fig. 8 where sEMG electrode also records cardiac pulses with different shapes. M3 efficiently detects and removes cardiac pulses, leaving intact not interfering parts of the signals. This method limits the damage to the signal of interest and the errors of signals interpretation.

In order to evaluate $M 3$ with real data, twelve healthy volunteers with mean age of $23.52 \pm 3.1$ years were subjected to four SMT force curves with different peak forces of $65,100,150$ and 225 N. Participants and applied forces are referred to $o=1,2, \ldots, 12$ and $i=1,2$, $\ldots, 4$. The correlation coefficients $\xi_{o, i, k}(13)$ of all subjects $o$, all SMT force curves $i$ and all electrodes $k$ are computed to compare performances of $M 1, M 2, M 3$ and $M 4$. This quantitative assessment, used in [13], is low when estimated EMG $\hat{s}_{o, i, k}^{\mathrm{EMG}}[n]$ and ECG parts $\hat{s}_{o, i, k}^{\mathrm{ECG}}[n]$ are uncorrelated, or supposed to be clearly separated.

$\xi_{o, i, k}=\frac{1}{N} \sum_{n=1}^{N} \hat{s}_{o, i, k}^{\mathrm{EMG}}[n] \hat{s}_{o, i, k}^{\mathrm{ECG}}[n]$

Fig. 9.a presents means of the correlation coefficients for all participants and applied forces and shows that $M 3$ reduces ECG more accurately than other methods. Indeed, a one-way repeated ANOVA was performed on obtained correlation coefficients and reports a significant effect on methods

$[\mathrm{F}(3,1161)=16.319, p=0.000025]$. Then, a Turkey post-hoc test reveals that $M 3$ presents correlation coefficients significantly lower than $M 1, M 2$ and $M 4(p=0.00008)$. Indeed, $M 1$ and $M 2$ cancel noiseless parts of signal. Identical to $M 3$ but with a less effective pulses detection method, $M 4$ returns a similar result than $M 1$ and $M 2$. The performance of $M 4$ can be explained with the figure Fig. $9 \mathrm{~b}$ where mean of correlation coefficients are presented for each applied peak force. Contrary to other methods, coefficients obtained for M4 increase with the level of peak force. As explained in the study exposed in [3], the higher the applied peak force is and the higher is the muscular activity response. The application of quick peak force creates muscular bursts (EMG) which is identified by the detection method of M4 as ECG pulsations. Even if signals present some EMG bursts, the proposed method M3 preserves the EMG information and rejects ECG pulsations. 

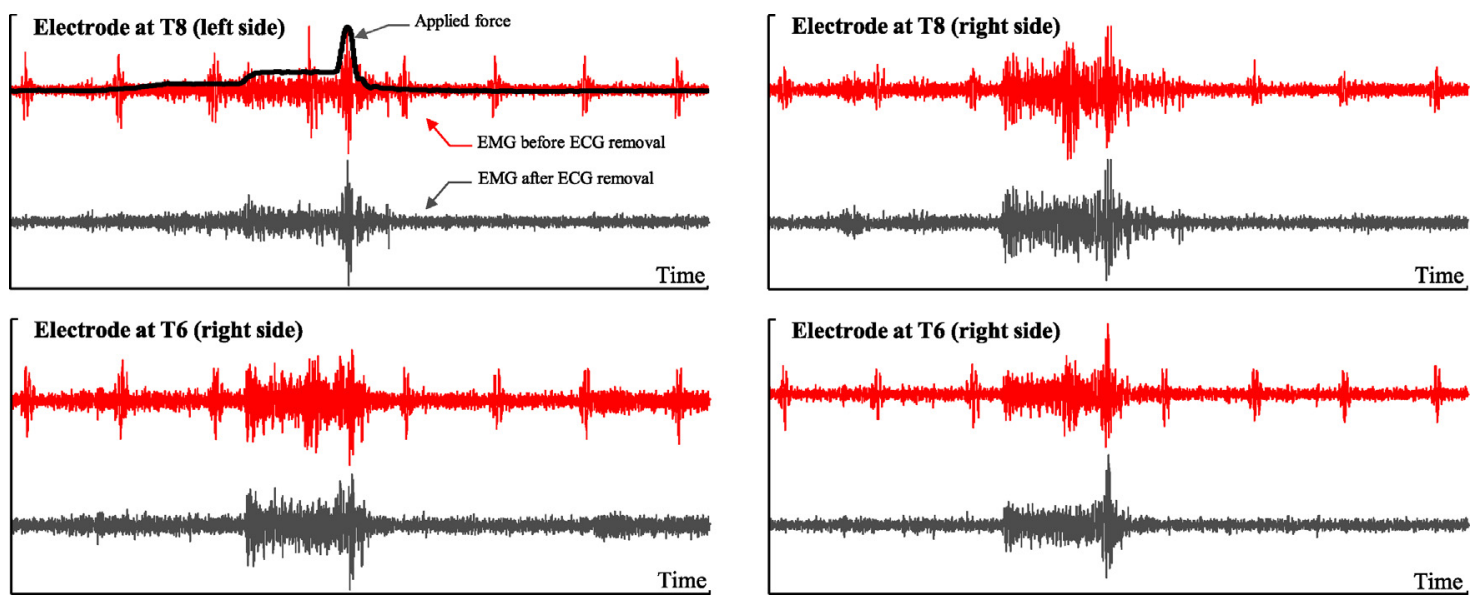

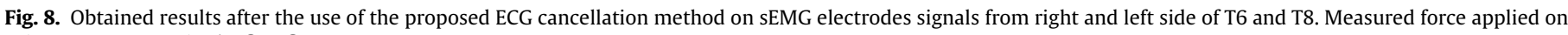
spine at $\mathrm{T} 7$ appears in the first figure.

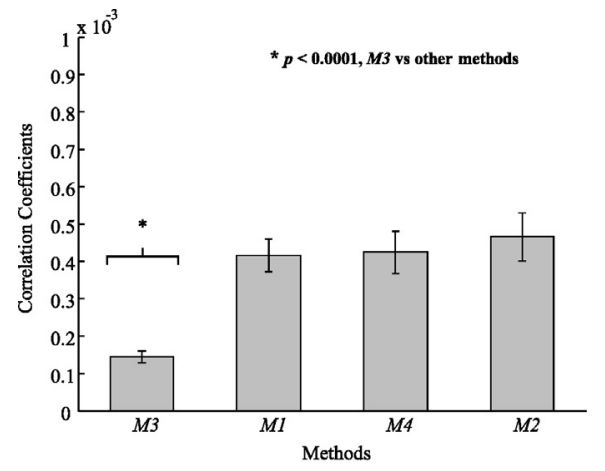

(a)

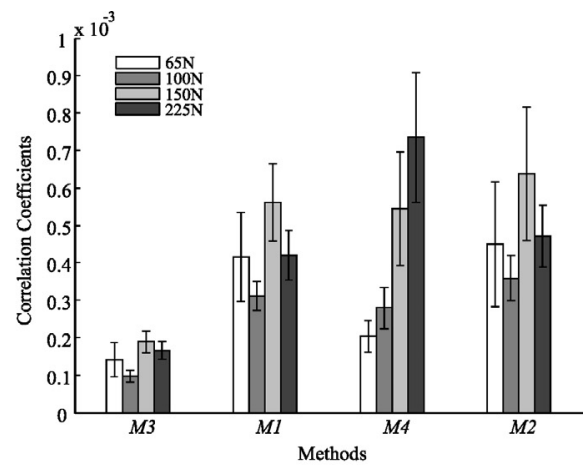

(b)

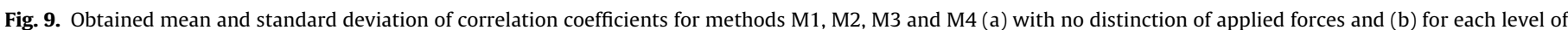
applied peak forces.

\section{Conclusion}

Muscular activities (EMG) recorded in the thoracic or trunk region are strongly contaminated by the cardiac pulsations (ECG), which limit the interpretations of the EMG signals, especially in case of low-amplitude responses. ECG artifacts must be rejected and the EMG preserved as much as possible. For this reason, the proposed method does not directly attempt to remove the ECG, but acts in two main phases: the localization of the ECG pulses and their cancellation only where they have been located. The localization phase uses a combination of DWT and ICA to efficiently extract ECG, and then takes advantage of its quasi-periodic nature to accurately detect pulses with a proposed method based on FFT.

The simulations, conducted in time- and frequency-domain for different levels of ECG contamination, and the evaluation from experimental data demonstrate that the proposed method efficiently cancels the ECG pulses from the EMG signal, even when they are more difficult to detect, and limits damages on the EMG in the noiseless parts of the signal. In addition, the beneficial impact of the local ECG cancellation, the combination of DWT and ICA, and the proposed pulses detection have clearly been shown.

Several research perspectives can be considered with this proposed method. Actually performed by a simple high pass filter, ECG cancellation phase could be improved. Pulses localization could be efficiently used in an adaptive structure, or with others techniques to realize local rejection. Note that, the overall structure of this method is adequate to apply an iterative process to refine the cancellation performances at each iteration. Finally, the pro- posed method with its efficient quasi-periodic signals detection could be introduced in other context characterized by the presence of periodic or quasi-signals for cancellation or detection.

\section{Acknowledgements}

This work is supported in part by the Fonds Excellence UQTR and the Natural Sciences and Engineering Research Council of Canada (NSERC).

\section{References}

[1] J.D. Drake, J.P. Callaghan, Elimination of electrocardiogram contamination from electromyogram signals: an evaluation of currently used removal techniques, J. Electromyogr. Kinesiol. 16 (2) (2006) 175-183 (2005).

[2] M. Descarreaux, F. Nougarou, C. Dugas, Standardization of spinal manipulation therapy in humans: development of a novel device designed to measure dose-response, J. Manipulative Physiol. Ther. 36 (2) (2013) 78-83.

[3] F. Nougarou, C. Deslauriers, C. Dugas, M. Descarreaux, Physiological responses to spinal manipulation therapy: investigation of the relationship between electromyographic responses and peak force, J. Manipulative Physiol. Ther. 39 (9) (2013) 557-563.

[4] F. Nougarou, C. Dugas, M. Loranger, I. Pagé, M. Descarreaux, The role of preload forces in spinal manipulation: experimental investigation of kinematic and electromyographic responses in healthy adults, J. Manipulative Physiol. Ther. 37 (5) (2014) 287-293.

[5] B.S. Darak, S.M. Hambrade, A review of techniques for extraction of cardiac artefacts in surface EMG signals and results for simulation of ECG-EMG mixture signal, in: IEEE International Conference on Pervasive Computing (ICPC), April 2015, 2015, pp. 1-5.

[6] N. Miljković, N. Popović, O. Djordjević, L. Konstantinović, T.B. Šekara, ECG artifact cancellation in surface EMG signals by fractional order calculus application, J. Comput. Methods Programs Biomed. 140 (2017) 256-264. 
[7] M.S. Redfern, R.E. Hughes, D.B. Chaffin, High-pass filtering to remove electrocardiographic interference from torso EMG recordings, Clin. Biomech. 8 (1) (1993) 44-48.

[8] M. Golabbakhsh, M. Masoumzadeh, M. Farzan Sabahi, ECG and power line noise removal from respiratory EMG signal using adaptive filters, Majlesi J. Electr. Eng. 5 (4) (2011) 28-33.

[9] G. Lu, S. Brittain, P. Hollan, J. Yianni, A.L. Green, F. Stein, T.Z. Aziz, S. Wang, Removing ECG noise from surface EMG signals using adaptive filtering, Neurosci. Lett. 462 (1) (2009) 14-19.

[10] S. Haykin, Adaptive Filter Theory, 4th ed., Prentice Hall, 2002.

[11] M.E.F. Djellatou, F. Nougarou, D. Massicotte, Enhanced FBLMS algorithm for ECG and noise removal from sEMG signals, in: IEEE 18th International Conference on Digital Signal Processing (DSP), 1-6, July, 2013.

[12] B. Widrow, J.R. Glover, Jr., J.M. McCool, J. Kaunitz, C.S. Williams, R.H. Hearn, et al., Adaptive noise cancelling: principles and applications, Proc. IEEE 63 (12) (1975) 1692-1716.

[13] S. Sanei, T.K.M. Lee, V. Abolghasemi, A new adaptive line enhancer based on singular spectrum analysis, IEEE Trans. Biomed. Eng. 59 (February (2)) (2012) 428-434.

[14] S. Sanei, A.R. Hosseini-Yazdi, Extraction of ECG from single channel EMG signal using constrained singular spectrum analysis, in: IEEE 17th International Conference on Digital Signal Processing (DSP), 1-4, July 2011, 2011.

[15] Y. Hu, X. Li, X. Xie, L. Pang, Y. Cao, K. Luk, Applying independent component analysis on ECG cancellation technique for the surface recording of trunk electromyography, Annual International Conference of IEEE Engineering in Medicine and Biology Society (2005) 3647-3649.

[16] N. Golyandina, V. Nekrutkin, A. Zhigljavsky, Analysis of Time Series Structure: SSA and Related Techniques, Chapman \& Hall/CRC, New York, 2001.

[17] J.N.F. Mak, Y. Hu, K.D.K. Luk, An automated ECG-artifact method for trunk muscle surface EMG recordings, J. Med. Eng. Phys. 32 (2010) 840-848.

[18] J.N.F. Mak, Y. Hu, K. Luk, ICA-based ECG removal from surface electromyography and its effect on low back pain assessment, IEEE EMBS Conf. Neural Eng. (2007) 646-649.

[19] J.D. Costa jr, D.D. Ferreira, A.M.F.L. Miranda de Sá, Reducing electrocardiographic artifacts from electromyogram signals with Independent Component Analysis, in: IEEE EMBS International Conference, September 2010, 2017, pp. 4598-4601.

[20] Y. Hu, J.N.F. Mak, K.D.K. Luk, Effect of electrocardiographic contamination on surface electromyography assessment of back muscles, J. Electromyogr. Kinesiol. 19 (1) (2009) 145-156.

[21] S.R. Alty, W.D.C. Man, J. Moxham, K.C. Lee, Denoising of diaphragm signals for respiratory control and diagnostic purposes, Annual International Conference of IEEE Engineering in Medicine and Biology Society (2008) 5560-5563.
[22] Y. Hu, J. Mak, H. Liu, K.D.K. Luk, ECG cancellation for surface electromyography measurement using independent component analysis, IEEE Int. ISCAS (2007) 3235-3238.

[23] S. Sanei, M. Ghodsi, H. Hassani, An adaptive singular spectrum analysis approach to murmur detection from heart sounds, J. Med. Eng. Phys. 33 (3) (2011) 362-367.

[24] J. Barrios-Muriel, F. Romero, F.J. Alonso, K. Gianikellis, A simple SSA-based de-noising technique to remove ECG interference in EMG signals, J. Biomed. Signal Process. Control 30 (2016) 1117-1126.

[25] M.E.F. Djellatou, D. Massicotte, M. Boukadoum, Adaptive block SSA based ANC implementation for high performances ECG removal from sEMG signals, in: IEEE 27th Canadian Conference on Electrical and Computer Engineering (CCECE), May 2014, 2014, pp. 1-6.

[26] F. Nougarou, D. Massicotte, M. Descarreaux, Detection method of flexion-relaxation phenomenon based on wavelets for patients with low back pain, EURASIP J. Adv. Signal Process. (2012) 1-17.

[27] A.M. Subasi. Yilmaz, H.R. Ozcalik, Classification of EMG signal using wavelet neural, J. Neurosci. Methods 156 (September (1-2)) (2006) 360-367.

[28] S. Mallat, A Wavelet Tour of Signal Processing, Academic Press, 1998.

[29] A. Medl, Time frequency and wavelets in biomedical signal processing, IEEE Eng. Med. Biol. Mag. 17 (December (6)) (1998).

[30] F. Nougarou, D. Massicotte, M. Descarreaux, Efficient combination of DWT and ICA to localize and remove ECG from surface electromyography measurement, in: IEEE International Conference on Digital Signal Processing (DSP), July, 2013.

[31] S. Abbaspour, M. Linden, H. Gholamhosseini, ECG artifact removal from surface EMG signal using an automated method based on wavelet-ICA, Stud. Health Technol. Inf. 211 (2015) 91-97.

[32] S. Abbaspour, A. Fallah, M. Linden, H. Gholamhosseini, A novel approach for removing ECG interferences from surface EMG signals using a combined ANFIS and wavelet, J. Electromyogr. Kinesiol. 26 (2016) 52-59.

[33] J. Taelman, S. Van Huffel, A. Spaepen, Wavelet-independent component analysis to remove electro-cardiography contamination in surface electromyography, Annual International Conference of IEEE Engineering in Medicine and Biology Society (2007) 682-685.

[34] V. von Tscharner, B. Eskofier, P. Federolf, Removal of the electrocardiogram signal from surface EMG recordings using nonlinearly scaled wavelets, J. Electromyogr. Kinesiol. 21 (4) (2011) 683-688.

[35] S.S. Manikandan, K.P. Soman, A novel method for detecting R peaks in electrocardiogram, J. Biomed. Signal Process. Control 7 (2) (2011) 118-128. 